\title{
General Spectral Flow Formula for Fixed Maximal Domain
}

\author{
Bernhelm Booss-Bavnbek and Chaofeng Zhu
}

\begin{abstract}
We consider a continuous curve of linear elliptic formally self-adjoint differential operators of first order with smooth coefficients over a compact Riemannian manifold with boundary together with a continuous curve of global elliptic boundary value problems. We express the spectral flow of the resulting continuous family of (unbounded) self-adjoint Fredholm operators in terms of the Maslov index of two related curves of Lagrangian spaces. One curve is given by the varying domains, the other by the Cauchy data spaces. We provide rigorous definitions of the underlying concepts of spectral theory and symplectic analysis and give a full (and surprisingly short) proof of our General Spectral Flow Formula for the case of fixed maximal domain. As a side result, we establish local stability of weak inner unique continuation property (UCP) and explain its role for parameter dependent spectral theory.
\end{abstract}

\section{Statement of the problem and main result}

1.1. Statement of the problem. Roughly speaking, the spectral flow counts the net number of eigenvalues changing from the negative real half axis to the non-negative one. The definition goes back to a famous paper by M. Atiyah, V. Patodi, and I. Singer [3, and was made rigorous by J. Phillips 23 for continuous paths of bounded selfadjoint Fredholm operators, by K.P. Wojciechowski [29] and C. Zhu

2000 Mathematics Subject Classification. Primary 58J30; Secondary 53D12.

Key words and phrases. Spectral flow, Maslov index, elliptic boundary value problems.

This work was supported in part by The Danish Science Research Council, SNF grant 21-02-0446. The second author is partially supported by FANEDD 200215, 973 Program of MOST, Fok Ying Tung Edu. Funds 91002, LPMC of MOE of China, and Nankai University. 
and Y. Long 33 in various non-self-adjoint cases, and by B. BoossBavnbek, M. Lesch, and J. Phillips [7 in the unbounded self-adjoint case. We shall give a rigorous definition of spectral flow, most suitable for our purpose, below in Subsection 2.1 together with a review of its basic properties. For a definition of spectral flow admitting zero in the continuous spectrum, we refer to A. Carey and J. Phillips [13].

In various branches of mathematics one is interested in the calculation of the spectral flow of a continuous family of closed densely defined (not necessarily bounded) self-adjoint Fredholm operators in a fixed Hilbert space. We consider the following typical problem of this kind.

Assumption 1.1. Let $\left\{A_{s}: C^{\infty}(M ; E) \rightarrow C^{\infty}(M ; E)\right\}_{s \in[0,1]}$ be a family of formally self-adjoint linear elliptic differential operators of first order with continuously varying smooth coefficients over a smooth compact Riemannian manifold $M$ with boundary $\Sigma$, acting on sections of a Hermitian vector bundle $E$ over $M$. Let $\left\{P_{s}\right\}$ be a continuous family of orthogonal pseudodifferential projections in $L^{2}\left(\Sigma ;\left.E\right|_{\Sigma}\right)$. Define $A_{s, P_{s}}$ to be the unbounded operator in $L^{2}(M ; E)$ with domain

$$
D_{s}:=\left\{x \in H^{1}(M ; E) \mid P_{s}(\gamma(x))=0\right\},
$$

where

$$
\gamma: H^{1}(M ; E) \rightarrow H^{\frac{1}{2}}\left(\Sigma ;\left.E\right|_{\Sigma}\right)
$$

denotes the (continuous) trace map from the first Sobolev space over the whole manifold to the $\frac{1}{2}$ Sobolev space over the boundary. (Note that in this paper the symbols $x$ and $y$ do not denote points of the underlying manifolds $M$ or $\Sigma$, but points in Hilbert spaces, sections of vector bundles, etc., following the conventions of functional analysis and dynamical systems.) Assume that each $P_{s}$ defines a self-adjoint elliptic boundary condition for $A_{s}$, i.e., $A_{s, P_{s}}$ is a self-adjoint Fredholm operator for each $s \in[0,1]$.

Then the spectral flow $\operatorname{sf}\left\{A_{s, P_{s}} ; s \in[0,1]\right\}$ or, shortly, $\operatorname{sf}\left\{A_{s, P_{s}}\right\}$ is well defined. As a spectral invariant it is essentially a quantum variable which one may not always be able to determine directly by eigenvalue calculations. As an alternative, one is looking for a classical method of calculating the spectral flow. There are two different approaches. One setting expresses the spectral flow (of a loop of Dirac operators on a closed manifold) as an integral over a 1-form induced by the heat kernel (for a review see [13]). The other setting is reduction to the boundary, i.e., one expresses the spectral flow (of a path of self-adjoint boundary value problems on a compact manifold with boundary) in terms of the 
intersection geometry of the solution spaces of the homogeneous differential equations and the boundary conditions. That is the approach we shall follow in this paper.

Problem 1.2. Give a classical method of calculating the spectral flow of the family $\left\{A_{s, P_{s}}\right\}$ by reduction to the boundary, i.e., a method not involving the determination of the spectrum near 0 and yielding an expression on $\Sigma$.

The preceding spectral flow calculation problem is formulated for families by analogy with Bojarski's Theorem for single operators which expresses the index (which is the difference between the multiplicities of the 0-eigenvalue of the original and the formally adjoint problem and so a priori a quantum or spectral invariant) of an elliptic operator over a closed partitioned manifold $M=M_{-} \cup_{\Sigma} M_{+}$by the index of the Fredholm pair of Cauchy data spaces from two sides along the hypersurface $\Sigma$ (which are classical objects, see Bojarski 4 and Booss and Wojciechowski [10. Chapter 24]).

1.2. General functional analytic setting and announcement of the General Spectral Flow Formula. Now we translate our problem into a functional analytic setting. For any such family there are three geometrically defined relevant Hilbert spaces of global sections which remain fixed under variation of the coefficients of the operators and under variation of the boundary conditions:

$$
L^{2}(M ; E), \quad H_{0}^{1}(M ; E), \quad \text { and } H^{1}(M ; E) .
$$

Here $H_{0}^{1}(M ; E)$ denotes the closure of $C_{0}^{\infty}(M \backslash \Sigma ; E)$ in the first Sobolev space $H^{1}(M ; E)$, where $C_{0}^{\infty}(M \backslash \Sigma ; E)$ denotes the smooth sections with support in the interior of $M \backslash \Sigma$. Since the trace map $\gamma: H^{1}(M ; E) \rightarrow$ $H^{\frac{1}{2}}\left(\Sigma ;\left.E\right|_{\Sigma}\right)$ is continuous, we have $H_{0}^{1}(M ; E)=\operatorname{ker} \gamma$, i.e., the space $H_{0}^{1}(M ; E)$ consists exactly of the elements of $H^{1}(M ; E)$ which vanish on the boundary $\Sigma$.

For each $s \in[0,1]$, we shall denote the unbounded operator $A_{s}$ acting in $L^{2}(M ; E)$ with domain $H_{0}^{1}(M ; E)$ also by $A_{s}$. Since the differential operator $A_{s}$ is elliptic, the unbounded operator $A_{s}$ is closed by Gårding's inequality

$$
\|x\|_{H^{1}(M ; E)} \leq C\left(\|x\|_{L^{2}(M ; E)}+\left\|A_{s} x\right\|_{L^{2}(M ; E)}\right) \quad \text { for } x \in H_{0}^{1}(M ; E) \text {. }
$$

Denote by $\operatorname{dom}(A)$ the domain of an operator $A$, by $A^{*}$ the adjoint operator of $A$, and

$$
D_{\max }(A):=\operatorname{dom}\left(A^{*}\right)
$$


Since $A$ is closed and symmetric, it follows that $D_{\max }(A)=\{x \in$ $\left.L^{2}(M ; E) \mid A x \in L^{2}(M ; E)\right\}$ with $A x$ taken in the distributional sense. For $A_{s}$ formally self-adjoint, it follows immediately that $H^{1}(M ; E) \subset$ $D_{\max }\left(A_{s}\right)$ and that $A_{s}$ (with domain $H_{0}^{1}(M ; E)$ ) is symmetric.

In local coordinates, we view each coefficient of $A_{s}$ as a map which maps $s \in[0,1]$ to a continuous section (which is actually smooth). Then the continuity of the curve $\left\{A_{s}\right\}_{s \in[0,1]}$ in the sense of continuously varying coefficients implies the continuity of the curve

$$
\left.[0,1] \ni s \mapsto A_{s}^{*}\right|_{H^{1}(M ; E)} \in \mathcal{B}\left(H^{1}(M ; E), L^{2}(M ; E)\right),
$$

as a curve of bounded operators from $H^{1}(M ; E)$ to $L^{2}(M ; E)$.

We denote by $Q_{s}: L^{2}\left(\Sigma ;\left.E\right|_{\Sigma}\right) \rightarrow L^{2}\left(\Sigma ;\left.E\right|_{\Sigma}\right)$ the Calderón projection. It is a projection onto the Cauchy data space of $A_{s}^{*}$ which is defined as the $L^{2}$-closure of $\gamma\left(\operatorname{ker}\left(\left.A_{s}^{*}\right|_{H^{1}(M ; E)}\right)\right.$. It can be described as a pseudodifferential operator, e.g., when continuing $A_{s}$ to an elliptic operator on a closed manifold $\widetilde{M} \supset M$, see R.T. Seeley [28, Sections 4 and 8] and [10, Chapter 12]. For an alternative canonical construction based on a natural boundary value problem and avoiding the choices of closing the manifold and continuing the operator, see B. Himpel, P. Kirk, and M. Lesch [16. Section 3] and recent joint work of the authors with M. Lesch 8 .

For each $s \in[0,1]$, there is a natural (strong) symplectic form $\omega_{s}$ on the quotient space $D_{\max }\left(A_{s}\right) / H_{0}^{1}(M ; E)$ induced by Green's form of $A_{s}$ as

$$
\omega_{s}(\gamma(x), \gamma(y)):=\left\langle A_{s}^{*} x, y\right\rangle-\left\langle x, A_{s}^{*} y\right\rangle, \quad x, y \in D_{\max }\left(A_{s}\right) .
$$

Here $\gamma$ denotes the natural projection

$$
D_{\max }\left(A_{s}\right) \rightarrow D_{\max }\left(A_{s}\right) / H_{0}^{1}(M ; E) .
$$

Identifying the quotient space $D_{\max }\left(A_{s}\right) / H_{0}^{1}(M ; E)$ with a subspace of the Sobolev (distribution) space $H^{-1 / 2}\left(\Sigma ;\left.E\right|_{\Sigma}\right)$, we obtain that this $\gamma$ extends the Sobolev trace map of (1.2). A rigorous definition of symplectic structures and Lagrangian subspaces will be given below in Subsection 2.2.

For our formally self-adjoint differential operators of first order, we have an explicit description of the form in (1.7), restricted to $H^{1}(M ; E)$, by Stokes' Theorem

$$
\omega_{s}(\gamma(x), \gamma(y))=-\int_{\Sigma}\left\langle\sigma_{1}\left(A_{s}\right)(\cdot, d t)\left(\left.x\right|_{\Sigma}\right),\left.y\right|_{\Sigma}\right\rangle d \mathrm{vol}_{\Sigma},
$$

where $\sigma_{1}\left(A_{s}\right)(\cdot, d t)$ denotes the principal symbol of $A_{s}$ at the boundary, taken in inner (co-)normal direction $d t$. Notice that we do not require 
that the manifold $M$ is orientable: for our application of Stokes' Theorem it suffices that any collar neighborhood of $\Sigma$ in $M$ is oriented by the normal structure. Then the form $\left.\omega_{s}\right|_{H^{1}(M ; E)}$ of (1.8) extends to a (strong) symplectic structure $\bar{\omega}_{s}$ on $L^{2}\left(\Sigma ;\left.E\right|_{\Sigma}\right)$. One can show that $\left.\omega_{s}\right|_{H^{1}(M ; E) / H_{0}^{1}(M ; E)}$ is a weak (but not strong) symplectic form on the Hilbert space $H^{1}(M ; E) / H_{0}^{1}(M ; E) \cong H^{\frac{1}{2}}\left(\Sigma ;\left.E\right|_{\Sigma}\right)$ (cf. Booss and Zhu 11. Remark 1.6b]).

We have $H^{1}(M ; E)=D_{\max }\left(A_{s}\right)$ if and only if $\operatorname{dim} M=1$. For higher dimensional case, the strict inclusion $H^{1}(M ; E) \subset D_{\max }\left(A_{s}\right)$ and the weakness of $\left.\omega_{s}\right|_{H^{1}(M ; E)}$ causes technical difficulties.

However, we still have the following theorem (cf. Theorem 0.1 of $11)$.

Theorem 1.3 (General Spectral Flow Formula). Let $\left\{A_{s}\right\}_{s \in[0,1]}$ and $\left\{P_{s}\right\}_{s \in[0,1]}$ be operator families like in Assumption 1.1. We assume that $\left\{\operatorname{ker} P_{s}\right\}_{s \in[0,1]}$ is a continuous family of Lagrangian subspaces in $\left(H, \bar{\omega}_{s}\right)$. If $A_{s}$ satisfies weak inner $U C P$, i.e., ker $A_{s}=\{0\}$ for each $s \in[0,1]$, we have:

(a) The family $\left\{A_{s, P_{s}}\right\}_{s \in[0,1]}$ of closed self-adjoint Fredholm operators on $X$ is a continuous family (in the gap norm, or equivalently, in the projection norm).

(b) The Cauchy data spaces $\operatorname{im} Q_{s}$ are Lagrangian subspaces in the weak symplectic Hilbert space $\left(H^{\frac{1}{2}}\left(\Sigma ;\left.E\right|_{\Sigma}\right), \bar{\omega}_{s}\right)$ and form a continuous family in $H^{\frac{1}{2}}\left(\Sigma ;\left.E\right|_{\Sigma}\right)$ for $s \in[0,1]$.

(c) Finally, the following formula holds:

$$
\operatorname{sf}\left\{A_{s, P_{s}}\right\}=-\operatorname{Mas}\left\{\operatorname{ker} P_{s}, \operatorname{im} Q_{s}\right\},
$$

where the spectral flow sf and the Maslov index Mas are defined by Definitions 2.1 and 2.11 below respectively.

REMARK 1.4. (a) The General Spectral Flow Formula contains and generalizes all previously known spectral flow formulae, as given by M. Morse 21, W. Ambrose 1, J.J. Duistermaat 14, A. Floer 15, P. Piccione and D.V. Tausk [24] and [25, and C. Zhu [31] and 32] for the 1-dimensional setting of the study of geodesics, and for the higher dimensional setting the formulae given by T. Yoshida [30, L. Nicolaescu 22, S.E. Cappell, R. Lee, and E.Y. Miller [12], B. Booss, K. Furutani, and N. Otsuki [5] and [6], and P. Kirk and M. Lesch [18].

(b) The main difference to [5] and [6] is that we admit varying maximal domain and varying Fredholm domain. The main difference to 18 is that we admit more general operators than Dirac type operators with constant coefficients in normal direction close to the boundary. 
(c) The proof of the above theorem is rather technical and complicated. In this review article, we only prove the following fixed maximal domain case which completely covers all above cited one-dimensional cases (cf. Corollary 2.14 in [11]). Moreover, it contains [5] and [6] and generalizes it to varying Fredholm domains, and contains [18 for the case of fixed maximal domain and generalizes it under that restriction to more general operator families.

1.3. Statement of the result for fixed maximal domain. Let $X$ be a Hilbert space, and $D_{m} \subset D_{\text {max }}$ be two dense linear subspaces of $X$. Let $\left\{A_{s}\right\}_{s \in[0,1]}$ be a family of symmetric densely defined operators in $\mathcal{C}(X)$ with domain $\operatorname{dom}\left(A_{s}\right)=D_{m}$. Here we denote by $\mathcal{C}(X)$ all closed operators in $X$. Assume that $\operatorname{dom}\left(A_{s}^{*}\right)=D_{\max }$, i.e., the domain of the maximal symmetric extension $A_{s}^{*}$ of $A_{s}$ is independent of $s$.

We recall from [5] (see also B. Lawruk, J. Śniatycki, and W.M. Tulczyjew 19] for early investigation of symplectic structures and boundary value problems) for each $s \in[0,1]$ :

(i) The space $D_{\max }$ is a Hilbert space with the graph inner product

$$
\langle x, y\rangle_{\mathfrak{G}_{s}}:=\langle x, y\rangle_{X}+\left\langle A_{s}^{*} x, A_{s}^{*} y\right\rangle_{X} \quad \text { for } x, y \in D_{\max } .
$$

(ii) The space $D_{m}$ is a closed subspace in the graph norm and the quotient space $D_{\max } / D_{m}$ is a strong symplectic Hilbert space with the (bounded) symplectic form induced by Green's form

(1.11) $\omega_{s}\left(x+D_{m}, y+D_{m}\right):=\left\langle A_{s}^{*} x, y\right\rangle_{X}-\left\langle x, A_{s}^{*} y\right\rangle_{X} \quad$ for $x, y \in D_{\max }$.

(iii) If $A_{s}$ admits a self-adjoint Fredholm extension $A_{s, D_{s}}:=\left.A_{s}^{*}\right|_{D_{s}}$ with domain $D_{s}$, then the natural Cauchy data space (ker $A_{s}^{*}+$ $\left.D_{m}\right) / D_{m}$ is a Lagrangian subspace of $\left(D_{\max } / D_{m}, \omega_{s}\right)$.

(iv) Moreover, self-adjoint Fredholm extensions are characterized by the property of the domain $D_{s}$ that $\left(D_{s}+D_{m}\right) / D_{m}$ is a Lagrangian subspace of $\left(D_{\max } / D_{m}, \omega_{s}\right)$ and forms a Fredholm pair with $\left(\operatorname{ker} A_{s}^{*}+D_{m}\right) / D_{m}$.

(v) We denote the natural projection (which is independent of $s$ ) by

$$
\gamma: D_{\max } \longrightarrow D_{\max } / D_{m}
$$

The main result of this paper is the following theorem which reproves parts of the preceding list.

TheOREm 1.5 (General Spectral Flow Formula for fixed maximal domain). We assume that on $D_{\max }$ the graph norms induced by $A_{s}$, $0 \leq s \leq 1$ are mutually equivalent. Then we fix a graph norm $\mathcal{G}$ on 
$D_{\max }$ induced by $A_{0}$. Assume that $\left\{A_{s}^{*}: D_{\max } \rightarrow X\right\}$ is a continuous family of bounded operators and each $A_{s}$ is injective. Let $\left\{D_{s} / D_{m}\right\}$ be a continuous family of Lagrangian subspaces of $\left(D_{\max } / D_{m}, \omega_{s}\right)$, such that each $A_{s, D_{s}}$ is a Fredholm operator. Then:

(a) Each $\left(D_{s} / D_{m}, \gamma\left(\operatorname{ker}\left(A_{s}^{*}\right)\right)\right.$ is a Fredholm pair in $D_{\max } / D_{m}$.

(b) Each Cauchy data space $\gamma\left(\operatorname{ker} A_{s}^{*}\right)$ is a Lagrangian subspace of $\left(D_{\max } / D_{m}, \omega_{s}\right)$.

(c) The family $\left\{\gamma\left(\operatorname{ker} A_{s}^{*}\right)\right\}$ is a continuous family in $D_{\max } / D_{m}$.

(d) The family $\left\{A_{s, D_{s}}\right\}$ is a continuous family of self-adjoint Fredholm operators in $\mathcal{C}(X)$.

(e) Finally, we have

$$
\operatorname{sf}\left\{A_{s, D_{s}}\right\}=-\operatorname{Mas}\left\{\gamma\left(D_{s}\right), \gamma\left(\operatorname{ker} A_{s}^{*}\right)\right\}
$$

Acknowledgement. The first author thanks the organizers Jan Kubarski, Tomasz Rybicki, and Robert Wolak of the 6th Conference on Geometry and Topology of Manifolds (Krynica, Poland, May 2-8, 2004) for the opportunity to present the main ideas and various ramifications of this paper in a mini-course of four hours. We both thank the referee for corrections, thoughtful comments, and helpful suggestions which led to many improvements. The referee clearly went beyond the call of duty, and we are indebted.

\section{Definition of spectral flow and Maslov index}

2.1. Spectral flow, revisited and generalized. Let $X$ be a Hilbert space. For a self-adjoint Fredholm operator $A \in \mathcal{C}(X)$, there exists a unique orthogonal decomposition

$$
X=X^{+}(A) \oplus X^{0}(A) \oplus X^{-}(A)
$$

such that $X^{+}(A), X^{0}(A)$ and $X^{-}(A)$ are invariant subspaces associated to $A$, and $\left.A\right|_{X^{+}(A)},\left.A\right|_{X^{0}(A)}$ and $\left.A\right|_{X^{-}(A)}$ are positive definite, zero and negative definite respectively. We introduce vanishing, natural, or infinite numbers

$$
m^{+}(A):=\operatorname{dim} X^{+}(A), m^{0}(A):=\operatorname{dim} X^{0}(A), m^{-}(A):=\operatorname{dim} X^{-}(A),
$$

and call them Morse positive index, nullity and Morse index of $A$ respectively. For finite-dimensional $X$, the signature of $A$ is defined by $\operatorname{sign}(A)=m^{+}(A)-m^{-}(A)$ which yields an integer. The APS projection $Q_{A}$ (where APS stands for Atiyah-Patodi-Singer) is defined by

$$
Q_{A}\left(x^{+}+x^{0}+x^{-}\right):=x^{+}+x^{0}
$$

for all $x^{+} \in X^{+}(A), x_{0} \in X^{0}(A), x^{-} \in X^{-}(A)$. 
Let $\left\{A_{s}\right\}, 0 \leq s \leq 1$ be a continuous family of self-adjoint Fredholm operators. The spectral flow $\operatorname{sf}\left\{A_{s}\right\}$ of the family should be equal to $m^{-}\left(A_{0}\right)-m^{-}\left(A_{1}\right)$ if $\operatorname{dim} X<+\infty$. We will generalize this definition to general $X$.

For each $t \in[0,1]$, there exists a bounded open neighborhood $N_{t}$ of 0 such that $\partial N_{t}$ is of class $C^{1}, \sigma\left(A_{t}\right) \cap \partial N_{t}=\emptyset$, and $P\left(A_{t}, N_{t}\right)$ is a finite rank projection. Here we denote the spectrum of a closed operator $A$ by $\sigma(A)$, and the spectral projection by

$$
P(A, N):=-\frac{1}{2 \pi \sqrt{-1}} \int_{\partial N}(A-z I)^{-1} d z
$$

if $N$ is a bounded open subset of $\mathbb{C}$ with $C^{1}$ boundary and $\partial N \cap \sigma(A)=$ $\emptyset$. The orientation of $\partial N$ is chosen to make $N$ stay on the left side of $\partial N$. Since the family $\left\{A_{s}\right\}, 0 \leq s \leq 1$ is continuous, there exists a $\delta(t)>0$ for each $t \in[0,1]$ such that

$$
\sigma\left(A_{s}\right) \cap \partial N_{t}=\emptyset, \text { for all } s \in(t-\delta(t), t+\delta(t)) \cap[0,1] .
$$

Then

$$
\left\{P\left(A_{s}, N_{t}\right)\right\}_{s \in(t-\delta(t), t+\delta(t)) \cap[0,1]} \text { for fixed } t \in[0,1]
$$

is a continuous family of orthogonal projections. By Lemma I.4.10 in Kato [17, they have the same rank. We denote by $A(s, t)$ the operator $A_{s}$ acting on the finite-dimensional space $\operatorname{im} P\left(A_{s}, N_{t}\right)$. Since $[0,1]$ is compact, there exists a partition $0=s_{0}<\ldots<s_{n}=1$ and $t_{k} \in$ $\left[s_{k}, s_{k+1}\right], k=0, \ldots, n-1$ such that $\left[s_{k}, s_{k+1}\right] \subset\left(t_{k}-\delta\left(t_{k}\right), t_{k}+\delta\left(t_{k}\right)\right)$ for each $k=0, \ldots, n-1$.

Definition 2.1. The spectral flow $\operatorname{sf}\left\{A_{s}\right\}$ of the family $\left\{A_{s}\right\}, 0 \leq$ $s \leq 1$ is defined by

$$
\operatorname{sf}\left\{A_{s}\right\}:=\sum_{k=0}^{n-1}\left(m^{-}\left(A\left(s_{k}, t_{k}\right)\right)-m^{-}\left(A\left(s_{k+1}, t_{k}\right)\right)\right) .
$$

After carefully examining the above definition, inspired by [23], we find that the necessary data for defining any spectral flow are the following:

- a co-oriented bounded real 1-dimensional regular $C^{1}$ submanifold $\ell$ of $\mathbb{C}$ without boundary (we call such an $\ell$ admissible, and denote by $\ell \in \mathcal{A}(\mathbb{C})$ );

- a Banach space $X$;

- and a continuous family of admissible operators $A_{s}, 0 \leq s \leq 1$ in $\mathcal{A}_{\ell}(X)$. 
Here we define $A \in \mathcal{C}(X)$ to be admissible with respect to $\ell$, if there exists a bounded open neighborhood $N$ of $\ell$ in $\mathbb{C}$ with $C^{1}$ boundary $\partial N$ such that (i) $\partial N \cap \sigma(A)=\emptyset$; (ii) $N \cap \sigma(A) \subset \ell$ is a finite set; and (iii) $P_{\ell}^{0}(A):=P(A, N)$ is a finite rank projection.

Note that $P_{\ell}^{0}(A)$ does not depend on the specific choice of $N$. We call $\nu_{h, \ell}(A):=\operatorname{dimim} P_{\ell}^{0}(A)$ the hyperbolic nullity of $A$ with respect to $\ell$. We denote by $\mathcal{A}_{\ell}(X)$ the set of closed admissible operators with respect to $\ell$. It is an open subset of $\mathcal{C}(X)$.

Similarly as before, we can define the spectral flow $\operatorname{sf}_{\ell}\left\{A_{s}\right\}$. It counts the number of spectral lines of $A_{s}$ coming from the negative side of $\ell$ to the non-negative side of $\ell$. For the details, see [33].

EXAMPLES 2.2. a) In the above self-adjoint case, $\ell=\sqrt{-1}(-\epsilon, \epsilon)$ $(\epsilon>0)$ with co-orientation from left to right. Then a self-adjoint operator $A$ is admissible with respect to $\ell$ if and only if $A$ is Fredholm. b) Another important case is that $\ell=(1-\epsilon, 1+\epsilon)(\epsilon \in(0,1))$ with co-orientation from downward to upward, and all $A_{s}$ unitary. A unitary operator $A$ is admissible with respect to $\ell$ if and only if $A-I$ is Fredholm.

The spectral flow has the following properties (cf. 23] and Lemma 2.6 and Proposition 2.2 in 33 ).

Proposition 2.3. Let $\ell \in \mathcal{A}(\mathbb{C})$ be admissible and let $\left\{A_{s}\right\}, 0 \leq$ $s \leq 1$ be a curve in $\mathcal{A}_{\ell}(X)$. Then the spectral flow $\operatorname{sf}_{\ell}\left\{A_{s}\right\}$ is well defined, and the following holds:

(i) Catenation. Assume $t \in[0,1]$. Then we have

$$
\operatorname{sf}_{\ell}\left\{A_{s} ; 0 \leq s \leq t\right\}+\operatorname{sf}_{\ell}\left\{A_{s} ; t \leq s \leq 1\right\}=\operatorname{sf}_{\ell}\left\{A_{s} ; 0 \leq s \leq 1\right\} .
$$

(ii) Homotopy invariance. Let $A(s, t),(s, t) \in[0,1] \times[0,1]$ be a continuous family in $\mathcal{A}_{\ell}(X)$. Then we have

$$
\operatorname{sf}_{\ell}\{A(s, t) ;(s, t) \in \partial([0,1] \times[0,1])\}=0 .
$$

(iii) Endpoint dependence for Riesz continuity. Let $\mathcal{B}^{\text {sa }}(X)$, respectively $\mathcal{C}^{\mathrm{sa}}(X)$ denote the spaces of bounded, respectively closed self-adjoint operators in $X$. Let

$$
\begin{aligned}
R: \mathcal{C}^{\mathrm{sa}} & \rightarrow \mathcal{B}^{\mathrm{sa}}(X) \\
A & \mapsto A\left(A^{2}+I\right)^{-\frac{1}{2}}
\end{aligned}
$$

denote the Riesz transformation. Let $A_{s} \in \mathcal{C}^{\mathrm{sa}}(X)$ for $s \in$ $[0,1]$. Assume that $\left\{R\left(A_{s}\right)\right\}, 0 \leq s \leq 1$ is a continuous family. If $m^{-}\left(A_{0}\right)<+\infty$, then $m^{-}\left(A_{1}\right)<+\infty$ and we have

$$
\operatorname{sf}\left\{A_{s}\right\}=m^{-}\left(A_{0}\right)-m^{-}\left(A_{1}\right) \text {. }
$$


(iv) Product. Let $\left\{P_{s}\right\}$ be a curve of projections on $X$ such that $P_{s} A_{s} \subset A_{s} P_{s}$ for all $s \in[0,1]$. Set $Q_{s}=I-P_{s}$. Then we have $P_{s} A_{s} P_{s} \in \mathcal{A}_{\ell}\left(\operatorname{im} P_{s}\right) \subset \mathcal{C}\left(\operatorname{im} P_{s}\right), Q_{s} A_{s} Q_{s} \in \mathcal{A}_{\ell}\left(\operatorname{im} Q_{s}\right) \subset$ $\mathcal{C}\left(\operatorname{im} Q_{s}\right)$, and

$$
\operatorname{sf}_{\ell}\left\{A_{s}\right\}=\operatorname{sf}_{\ell}\left\{P_{s} A_{s} P_{s}\right\}+\operatorname{sf}_{\ell}\left\{Q_{s} A_{s} Q_{s}\right\} .
$$

(v) Bound. For $A \in \mathcal{A}_{\ell}(X)$, there exists a neighborhood $\mathcal{N}$ of $A$ in $\mathcal{C}(X)$ such that $\mathcal{N} \subset \mathcal{A}_{\ell}(X)$, and for curves $\left\{A_{s}\right\}$ in $\mathcal{N}$ with endpoints $A_{0}=: A$ and $A_{1}=: B$, the relative Morse index $I_{\ell}(A, B):=-\operatorname{sf}_{\ell}\left\{A_{s}, 0 ; \leq s \leq 1\right\}$ is well defined and satisfies

(vi) Reverse orientation. Let $\hat{\ell}$ denote the curve $\ell$ with opposite co-orientation. Then we have

$$
\operatorname{sf}_{\ell}\left\{A_{s}\right\}+\operatorname{sf}_{\hat{\ell}}\left\{A_{s}\right\}=\nu_{h, \ell}\left(A_{1}\right)-\nu_{h, \ell}\left(A_{0}\right) .
$$

(vii) Zero. Suppose that $\nu_{h, \ell}\left(A_{s}\right)$ is constant for $s \in[0,1]$. Then $\operatorname{sf}_{\ell}\left\{A_{s}\right\}=0$.

(viii) Invariance. Let $\left\{T_{s}\right\}_{s \in[0,1]}$ be a curve of bounded invertible operators. Then we have

$$
\operatorname{sf}_{\ell}\left\{T_{s}^{-1} A_{s} T_{s}\right\}=\operatorname{sf}_{\ell}\left\{A_{s}\right\}
$$

Now we give a method of calculating the spectral flow of differentiable curves, inspired among others by J.J. Duistermaat [14] and J. Robbin and D. Salamon [27].

Definition 2.4. Let $\ell \in \mathcal{A}(\mathbb{C})$ be admissible and $\left\{A_{s}\right\}_{s \in[0,1]}$ be a curve in $\mathcal{A}_{\ell}(X)$.

(i) A crossing for $A_{s}$ is a number $t \in[0,1]$ such that $\nu_{h, \ell}\left(A_{t}\right) \neq 0$.

(ii) Set $P_{s}=P_{\ell}^{0} A_{s}$. A crossing $t$ is called regular if $\operatorname{dom}\left(A_{s}\right)=D$ fixed for $s$ near $t, A_{s} x$ is differentiable at $s=t$ for all $x \in D$, and $P_{t} \dot{A}_{t} P_{t}$ is hyperbolic, i.e. $\nu_{h, \ell}\left(P_{t} \dot{A}_{t} P_{t}\right)=0$, where $\dot{A}_{s}$ is the unbounded operator with domain $D$ defined by

$$
\dot{A}_{s} x=\frac{d}{d s} A_{s} x
$$

for all $x \in D$.

(iii) A crossing $t$ is called simple if it is regular and $\nu_{h, \ell}\left(A_{t}\right)=1$.

Proposition 2.5 (cf. Theorem 4.1 of 33 ). Let $X$ be a Banach space and $\ell=\sqrt{-1}(-\epsilon, \epsilon)(\epsilon>0)$ with co-orientation from left to right. Let $A_{s},-\epsilon \leq s \leq \epsilon(\epsilon>0)$, be a curve in $\mathcal{A}_{\ell}(X)$. Suppose that 0 is 
a regular crossing of $A_{s}$. Set $P=P_{\ell}^{0}\left(A_{0}\right), A=A_{0}$ and $B=\left.\dot{A}_{s}\right|_{s=0}$. Assume that

$$
P(A B-B A) P=0 .
$$

Then there is a $\delta \in(0, \epsilon)$ such that $\nu_{h, \ell}\left(A_{s}\right)=0$ for all $s \in[-\delta, 0) \cup(0, \delta]$ and

$$
\begin{aligned}
& \operatorname{sf}_{\ell}\left\{A_{s} ; 0 \leq s \leq \delta\right\}=-m^{-}(P B P), \\
& \operatorname{sf}_{\ell}\left\{A_{s} ;-\delta \leq s \leq 0\right\}=m^{+}(P B P) .
\end{aligned}
$$

Here we denote by $m^{+}(P B P)\left(m^{-}(P B P)\right)$ the total algebraic multiplicity of eigenvalues of $P B P$ with positive (negative) imaginary part respectively.

2.2. Symplectic functional analysis and Maslov index. A main feature of symplectic analysis is the study of the Maslov index. It is an intersection index between a path of Lagrangian subspaces with the Maslov cycle, or, more generally, with another path of Lagrangian subspaces. The Maslov index assigns an integer to each continuous path of Fredholm pairs of Lagrangian subspaces of a fixed Hilbert space with continuously varying symplectic structures.

Firstly we define symplectic Hilbert spaces and Lagrangian subspaces.

Definition 2.6. Let $H$ be a complex vector space. A mapping

$$
\omega: H \times H \longrightarrow \mathbb{C}
$$

is called a (weak) symplectic form on $H$, if it is sesquilinear, skewhermitian, and non-degenerate, i.e.,

(i) $\omega(x, y)$ is linear in $x$ and conjugate linear in $y$;

(ii) $\omega(y, x)=-\overline{\omega(y, x)}$;

(iii) $H^{\omega}:=\{x \in H \mid \omega(x, y)=0$ for all $y \in H\}=\{0\}$.

Then we call $(H, \omega)$ a complex symplectic vector space.

Definition 2.7. Let $(H, \omega)$ be a complex symplectic vector space.

(a) The annihilator of a subspace $\lambda$ of $H$ is defined by

$$
\lambda^{\omega}:=\{y \in H \mid \omega(x, y)=0 \text { for all } x \in \lambda\} .
$$

(b) A subspace $\lambda$ is called isotropic, co-isotropic, or Lagrangian if

$$
\lambda \subset \lambda^{\omega}, \quad \lambda \supset \lambda^{\omega}, \quad \lambda=\lambda^{\omega},
$$

respectively.

(c) The Lagrangian Grassmannian $\mathcal{L}(H, \omega)$ consists of all Lagrangian subspaces of $(H, \omega)$. 
Definition 2.8. Let $H$ be a complex Hilbert space. A mapping $\omega: H \times H \rightarrow \mathbb{C}$ is called a (strong) symplectic form on $H$, if $\omega(x, y)=$ $\langle J x, y\rangle_{H}$ for some bounded invertible skew-adjoint operator $J$. $(H, \omega)$ is called a (strong) symplectic Hilbert space.

Before giving a rigorous definition of the Maslov index, we fix the terminology and give a simple lemma.

We recall:

Definition 2.9. (a) The space of (algebraic) Fredholm pairs of linear subspaces of a vector space $H$ is defined by (2.13)

$\mathcal{F}_{\text {alg }}^{2}(H):=\{(\lambda, \mu) \mid \operatorname{dim}(\lambda \cap \mu)<+\infty$ and $\operatorname{dim}(H /(\lambda+\mu))<+\infty\}$

with

$$
\operatorname{index}(\lambda, \mu):=\operatorname{dim}(\lambda \cap \mu)-\operatorname{dim}(H /(\lambda+\mu)) .
$$

(b) In a Banach space $H$, the space of (topological) Fredholm pairs is defined by

$$
\mathcal{F}^{2}(H):=\left\{(\lambda, \mu) \in \mathcal{F}_{\text {alg }}^{2}(H) \mid \lambda, \mu, \text { and } \lambda+\mu \subset H \text { closed }\right\} .
$$
$1.7])$.

We need the following well-known lemma (see, e.g., [11, Lemma

Lemma 2.10. Let $(H, \omega)$ be a (strong) symplectic Hilbert space. Then

(i) there is a 1-1 correspondence between the space

$$
\mathcal{U}^{J}=\left\{U \in \mathcal{B}\left(H^{+}, H^{-}\right)\left|U^{*} J\right|_{H^{-}} U=-\left.J\right|_{H^{+}}\right\}
$$

and $\mathcal{L}(H, \omega)$ under the mapping $U \rightarrow L:=\mathfrak{G}(U)$ (= graph of $U)$, where $H^{ \pm}=H^{\mp}(\sqrt{-1} J)$ in the sense of the decomposition (2.1);

(ii) if $U, V \in \mathcal{U}^{J}$ and $\lambda:=\mathfrak{G}(U), \mu:=\mathfrak{G}(V)$, then $(\lambda, \mu)$ is a Fredholm pair if and only if $U-V$, or, equivalently, $U V^{-1}-I$ is Fredholm. Moreover, we have a natural isomorphism

$$
\operatorname{ker}\left(U V^{-1}-I\right) \simeq \lambda \cap \mu .
$$

Definition 2.11. Let $\left(H,\langle\cdot, \cdot\rangle_{s}\right), s \in[0,1]$ be a continuous family of Hilbert spaces, and $\omega_{s}(x, y)=\left\langle J_{s} x, y\right\rangle_{s}$ be a continuous family of symplectic forms on $H$, i.e., $\left\{A_{s, 0}\right\}$ and $\left\{J_{s}\right\}$ are two continuous families of bounded invertible operators, where $A_{s, 0}$ is defined by

$$
\langle x, y\rangle_{s}=\left\langle A_{s, 0} x, y\right\rangle_{0} \quad \text { for all } x, y \in H .
$$


Let $\left\{\left(\lambda_{s}, \mu_{s}\right)\right\}$ be a continuous family of Fredholm pairs of Lagrangian subspaces of $\left(H,\langle\cdot, \cdot\rangle_{s}, \omega_{s}\right)$. Then there is a continuous splitting

$$
H=H_{s}^{-}\left(\sqrt{-1} J_{s}\right) \oplus H_{s}^{+}\left(\sqrt{-1} J_{s}\right)
$$

associated to the self-adjoint operator $\sqrt{-1} J_{s} \in \mathcal{B}\left(H,\langle\cdot, \cdot\rangle_{s}\right)$ for each $s \in[0,1]$. By Lemma 2.10, $\lambda_{s}=\mathfrak{G}_{s}\left(U_{s}\right)$ and $\mu_{s}=\mathfrak{G}_{s}\left(V_{s}\right)$ with $U_{s}$, $V_{s} \in \mathcal{U}^{J_{s}}$, where $\mathfrak{G}_{s}$ denotes the graph associated to the splitting (2.17). We define the Maslov index $\operatorname{Mas}\left\{\lambda_{s}, \mu_{s}\right\}$ by

$$
\operatorname{Mas}\left\{\lambda_{s}, \mu_{s}\right\}=-\operatorname{sf}_{\ell}\left\{U_{s} V_{s}^{-1}\right\},
$$

where $\ell:=(1-\epsilon, 1+\epsilon)$ with, $\epsilon \in(0,1)$ and with upward co-orientation.

REMARK 2.12. For finite-dimensional $H$, constant $\mu_{s}=\mu_{0}$, and a loop $\left\{\lambda_{s}\right\}$, i.e., for $\lambda_{0}=\lambda_{1}$, we notice that $\operatorname{Mas}\left\{\lambda_{s}, \mu_{s}\right\}$ is the winding number of the closed curve $\left\{\operatorname{det}\left(U_{s}^{-1} V_{0}\right)\right\}_{s \in[0,1]}$. This is the original definition of the Maslov index as explained in Arnol'd, 22.

LEMma 2.13. The Maslov index is independent of the choice of the complete inner product of $H$.

Proof. Let $\langle\cdot, \cdot\rangle_{s, k}, s \in[0,1]$ with $k=0,1$ be two continuous families of complete inner products of $H$. We define

$$
\langle\cdot, \cdot\rangle_{s, t}=(1-t)\langle\cdot, \cdot\rangle_{s, 0}+t\langle\cdot, \cdot\rangle_{s, 1}
$$

for each $(s, t) \in[0,1] \times[0,1]$. Let $\left(\lambda_{s}, \mu_{s}\right)$ be a continuous family of Fredholm pairs of Lagrangian subspaces of $\left(H, \omega_{s}\right)$. For each inner product $\langle\cdot, \cdot\rangle_{s, t}$, we denote by $U_{s, t}$ and $V_{s, t}$ the associated generated "unitary" operators of $\lambda_{s}$ and $\mu_{s}$ respectively. We also denote by Mas the Maslov index defined with $\langle\cdot, \cdot\rangle_{s, t}$ for each $t \in[0,1]$. By Proposition 2.3 we have

$$
\begin{aligned}
\operatorname{Mas}_{0}\left\{\lambda_{s}, \mu_{s}\right\} & -\operatorname{Mas}_{1}\left\{\lambda_{s}, \mu_{s}\right\} \\
& =-\operatorname{sf}_{\ell}\left\{U_{s, 0} V_{s, 0}^{-1}\right\}+\operatorname{sf}_{\ell}\left\{U_{s, 1} V_{s, 1}^{-1}\right\} \\
& =-\operatorname{sf}_{\ell}\left\{U_{s, t} V_{s, t}^{-1} ;(s, t) \in \partial([0,1] \times[0,1])\right\} \\
& =0 .
\end{aligned}
$$

Now we give a method of using the crossing form to calculate Maslov indices (cf. [14], 27], [5, Theorem 2.1]; for a full proof of the following Proposition see [32, Corollary 3.1]).

Let $\lambda=\left\{\lambda_{s}\right\}_{s \in[0,1]}$ be a $C^{1}$ curve of Lagrangian subspaces of $H$. Let $W$ be a fixed Lagrangian complement of $\lambda_{t}$. For $v \in \lambda_{t}$ and $|s-t|$ 
small, define $w(s) \in W$ by $v+w(s) \in \lambda_{s}$. The form

$$
Q(\lambda, t):=Q(\lambda, W, t)(u, v)=\left.\frac{d}{d s}\right|_{s=t} \omega(u, w(s)), \quad \forall u, v \in \lambda_{t}
$$

is independent of the choice of $W$. Let $\left\{\left(\lambda_{s}, \mu_{s}\right)\right\}, 0 \leq s \leq 1$ be a curve of Fredholm pairs of Lagrangian subspaces of $H$. For $t \in[0,1]$, the crossing form $\Gamma(\lambda, \mu, t)$ is a quadratic form on $\lambda_{t} \cap \mu_{t}$ defined by

$$
\Gamma(\lambda, \mu, t)(u, v)=Q(\lambda, t)(u, v)-Q(\mu, t)(u, v), \quad \forall u, v \in \lambda_{t} \cap \mu_{t}
$$

A crossing is a time $t \in[0,1]$ such that $\lambda_{t} \cap \mu_{t} \neq\{0\}$. A crossing is called regular if $\Gamma(\lambda, \mu, t)$ is nondegenerate. It is called simple if it is regular and $\lambda_{t} \cap \mu_{t}$ is one-dimensional.

Proposition 2.14. Let $(H, \omega)$ be a symplectic Hilbert space and $\left\{\left(\lambda_{s}, \mu_{s}\right)\right\}, 0 \leq s \leq 1$ be a $C^{1}$ curve of Fredholm pairs of Lagrangian subspaces of $H$ with only regular crossings. Then we have

$$
\operatorname{Mas}\{\lambda, \mu\}=m^{+}(\Gamma(\lambda, \mu, 0))-m^{-}(\Gamma(\lambda, \mu, 1))+\sum_{0<t<1} \operatorname{sign}(\Gamma(\lambda, \mu, t)) .
$$

\section{Symplectic analysis of symmetric operators}

3.1. Local stability of weak inner UCP. Let $X$ be a complex Hilbert space and $A \in \mathcal{C}(X)$ a linear, closed, densely defined operator in $X$. We assume that $A$ is symmetric, i.e., $A^{*} \supset A$ where $A^{*}$ denotes the adjoint operator. We denote the domains of $A$ by $D_{m}$ (the minimal domain) and of $A^{*}$ by $D_{\max }$ (the maximal domain).

Definition 3.1. Let $X$ be a Hilbert space and $A \in \mathcal{C}(X)$ with $\operatorname{dom} A=D_{m}$ and $A^{*} \supset A$. We shall say that the operator $A$ satisfies the weak inner Unique Continuation Property (UCP) if ker $A=\{0\}$.

It is well known that weak UCP and weak inner UCP can be established for a large class of Dirac type operators, see the first author with Wojciechowski [10. Chapter 8], and the first author with M. Marcolli and B.-L. Wang [9]. However, it is not valid for all linear elliptic differential operators of first order as shown by one of the Pliś counterexamples 26. Moreover, one has various quite elementary examples of linear and non-linear perturbations which invalidate weak inner UCP for Dirac operators. Two such examples are listed in $\mathbf{9}$. In the same paper, however, it was shown that weak UCP is preserved under certain 'small' perturbations of Dirac type operators. Here we show an elementary result, namely the local stability of weak inner UCP. 
Lemma 3.2. Let $X$ be a Hilbert space. Let $A_{s} \in \mathcal{C}(X), 0 \leq s \leq 1$ be a family of symmetric operators with $\operatorname{dom} A_{s}=D_{m}$ and $\operatorname{dom} A_{s}^{*}=$ $D_{\max }$ independent of $s$. Assume that $\left\{A_{s}^{*}: D_{\max } \rightarrow X\right\}$ is a continuous curve of bounded operators, where the norm on $D_{\max }$ is the graph norm induced by $A_{0}^{*}$. If $A_{0}$ satisfies weak inner UCP and there exists a selfadjoint Fredholm extension $\left.A_{0}^{*}\right|_{D}$ of $A_{0}$, then for all $s \ll 1$ the operators $A_{s}^{*}$ are surjective and the operators $A_{s}$ satisfy weak inner $U C P$.

Proof. By our assumptions, im $\left.A_{0}^{*}\right|_{D}$ is closed and is of finite codimension. Since $\left.\operatorname{im} A_{0}^{*}\right|_{D} \subset \operatorname{im} A_{0}^{*} \subset X$, the full range $\operatorname{im} A_{0}^{*}$ is closed. Since $A_{0}$ satisfies weak inner UCP, $\operatorname{im} A_{0}^{*}=X$. Then $A_{0}^{*}$ is semiFredholm. By Theorem IV.5.17 of Kato [17] we have $\operatorname{im} A_{s}^{*}=X$ for $s \ll 1$. Since $A_{s}$ are symmetric, $A_{s}$ satisfy weak inner UCP for $s \ll 1$.

3.2. Continuity of the family $\left\{A_{s, D_{s}}\right\}$. Let $X$ be a complex Hilbert space, and $M, N \subset X$ be two closed linear subspaces. Let $P_{M}, P_{N}$ be the orthogonal projections onto $M, N$ respectively. Then the distance $d(M, N)$ is defined by $d(M, N)=\left\|P_{M}-P_{N}\right\|$ and called the gap between $M$ and $N$. For any two closed operators $A, B$ on $X$, we define $d(A, B)$ as the distance between their graphs.

Let $A \in \mathcal{C}(X)$ be a linear, closed, densely defined operator in $X$. By Footnote 1 (page 198), Theorems IV.1.1 and IV.2.14 in [17, it is easy to verify the following

Lemma 3.3. Let $B \in \mathcal{B}(\operatorname{dom}(A), X)$ be a bounded operator, where the norm on $\operatorname{dom}(A)$ is the graph norm $\mathcal{G}_{A}$ induced by $A$. Let $d:=$ $\|B-A\|_{\mathcal{G}_{A}}<\frac{1}{2}$. Then we have

(i) $B \in \mathcal{C}(X)$, and it holds that

$$
(1-2 d)\langle x, x\rangle_{\mathcal{G}_{A}} \leq\langle x, x\rangle_{\mathcal{G}_{B}} \leq(1+d)^{2}\langle x, x\rangle_{\mathcal{G}_{A}} \text { for } x \in D
$$

(ii) $d(B, A) \leq \frac{\sqrt{2} d}{(1-d)^{-1}}$.

LEMma 3.4. Let $X$ be a Hilbert space, and $Y$ be a closed linear subspace of $H$. Then there exists a bijection between the space of closed linear subspaces of $X$ containing $Y$ and that of closed linear subspaces of $X / Y$ which preserves the metric.

Proof. We view $X / Y$ as $Y^{\perp}$. Let $M, N \subset Y^{\perp}$ be two closed subspaces and $P_{M}, P_{N}$ be the orthogonal projections onto $M, N$ respectively. Then we have

$$
d(M+Y, N+Y)=\left\|P_{M+Y}-P_{N+Y}\right\|=\left\|P_{M}-P_{N}\right\|=d(M, N) .
$$


From the definition of the gap norm and by some computations we have

Lemma 3.5. Let $D_{m} \subset D_{\max } \subset X$ be three Hilbert spaces such that $D_{m}$ is a closed subspace of $D_{\max }$ and a dense subspace of $X$. Let $\left\{A_{s} \in \mathcal{C}(X)\right\}_{s \in[0,1]}$ be a family of densely defined symmetric operators with domain $D_{m}$, and $\left\{D_{s}\right\}_{s \in[0,1]}$ be a family of closed subspaces of $D_{\max }$ containing $D_{m}$. We assume that $\operatorname{dom}\left(A_{s}^{*}\right)=D_{\max }$, each graph norm $\mathcal{G}_{s}$ of $D_{\max }$ induced by $A_{s}^{*}$ is equivalent to the original norm $\mathcal{G}$ of $D_{\max }$, and $\left\{A_{s}^{*} \in \mathcal{B}\left(D_{\max }, X\right)\right\},\left\{D_{s} / D_{m} \subset D_{\max } / D_{m}\right\}$ are two continuous families. Then $\left\{A_{s, D_{s}} \in \mathcal{C}(X)\right\}_{s \in[0,1]}$ is a continuous family of closed operators.

3.3. Continuity of natural Cauchy data spaces. In this subsection we generalize the proof of the continuity of Cauchy data spaces given in [5, Section 3.3]. We need the following

Proposition 3.6 (Proposition 3.5 of [5]). Let $X$ be a Hilbert space, and $A \in \mathcal{C}(X)$ be a symmetric operator. Set $D_{m}=\operatorname{dom}(A)$ and $D_{\max }=$ $\operatorname{dom}\left(A^{*}\right)$. If $A$ admits a self-adjoint Fredholm extension with domain $D$, then the quotient space $D / D_{m}$ and the natural Cauchy data space $\left(\operatorname{ker} A^{*}+D_{m}\right) / D_{m}$ form a Fredholm pair of Lagrangian subspaces of the (strong) symplectic Hilbert space $D_{\max } / D_{m}$ (introduced above in Subsection 1.3, Item $(i i))$.

REMARK 3.7. At present (March 2005), it is not known whether all linear formally self-adjoint elliptic differential operators of first order over a compact smooth Riemannian manifold with smooth boundary admit a self-adjoint Fredholm extension. Recently in [8, however, that crucial property has been established under the additional assumption of self-adjoint principal symbol of the "tangential operator" at the boundary.

Now we can prove

Proposition 3.8. Let $X$ be a Hilbert space, and $D_{m} \subset D_{\max }$ be two dense linear subspaces of $X$. Let $\left\{A_{s}: D_{m} \rightarrow X\right\}_{s \in[0,1]}$ be a family of closed symmetric densely defined operators in $X$. We assume that

(i) each $A_{s}$ admits a self-adjoint Fredholm extension with domain $D_{s}$

(ii) $\operatorname{dom}\left(A_{s}^{*}\right)=D_{\max }$ independent of $s$ and that all graph norms $\mathcal{G}_{s}$ of $D_{\max }$ induced by $A_{s}^{*}$ are mutually equivalent;

(iii) each $A_{s}$ satisfies weak inner $U C P$ relative to $D_{m}$; and 
(iv) $\left\{A_{s}^{*}: D_{\max } \rightarrow X\right\}$ forms a continuous family of bounded operators, where the norm on $D_{\max }$ is the graph norm $\mathcal{G}$ induced by $A_{0}$.

Then the natural Cauchy data spaces $\left(D_{m}+\mathrm{ker} A_{s}^{*}\right) / D_{m}$ are continuously varying in $D_{\max } / D_{m}$.

Proof. We denote the projection of $D_{\max }$ onto $D_{\max } / D_{m}$ by $\gamma$. Note that ker $A_{s}^{*}$ is closed in $D_{\max }$.

To prove the continuity, we need only to consider the local situation at $s=0$. First we show that $\left\{\operatorname{ker} A_{s}^{*}\right\}_{s \in[0,1]}$ is a continuous family of subspaces of $D_{\max }$; then we show that $\gamma\left(\operatorname{ker} A_{s}^{*}\right)$ is a continuous family in $D_{\max } / D_{m}$.

We consider the bounded operator

$$
\begin{array}{ccc}
F_{s}: & D_{\max } & \longrightarrow X \oplus \operatorname{ker} A_{0}^{*} \\
x & \mapsto\left(A_{s}^{*}(x), P_{0} x\right)
\end{array},
$$

where $P_{0}: D_{\max } \rightarrow \operatorname{ker} A_{0}^{*}$ denotes the orthogonal projection of the Hilbert space $D_{\max }$ onto the closed subspace ker $A_{0}^{*}$. By definition, the family $\left\{F_{s}\right\}$ is a continuous family of bounded operators.

Clearly, $F_{0}$ is injective. Since $\left.\operatorname{im} A_{0}^{*}\right|_{D_{0}} \subset \operatorname{im} A_{0}^{*} \subset X$ and $\left.A_{0}^{*}\right|_{D_{0}}$ is Fredholm, im $A_{0}^{*}$ is closed. From weak inner UCP we get $\operatorname{im} A_{0}^{*}=X$. So the operator $F_{0}$ is also surjective. This proves that $F_{0}$ is invertible with bounded inverse. Then all operators $F_{s}$ are invertible for small $s \geq 0$, since $F_{s}$ is a continuous family of operators.

Note that

$$
F_{s}\left(\operatorname{ker} A_{s}^{*}\right) \subset\{0\} \oplus \operatorname{ker} A_{0}^{*}, \quad\left(F_{s}\right)^{-1}\left(\{0\} \oplus \operatorname{ker} A_{0}^{*}\right) \subset \operatorname{ker} A_{s}^{*} .
$$

Since $F_{s}$ are invertible for small $s \geq 0$, we have

$$
F_{s}\left(\operatorname{ker} A_{s}^{*}\right)=\{0\} \oplus \operatorname{ker} A_{0}^{*} .
$$

We define

$$
\varphi_{s}:=F_{s}^{-1} \circ F_{0}: D_{\max } \cong D_{\max } \text { and } \varphi_{s}^{-1}=F_{0}^{-1} \circ F_{s}: D_{\max } \cong D_{\max }
$$

for $s$ small. Since $F_{s}$ are invertible for small $s \geq 0$, from (3.1) we obtain that

$$
\varphi_{s}\left(\operatorname{ker} A_{0}^{*}\right)=\operatorname{ker} A_{s}^{*} .
$$

From (3.2) we get that

$$
\left\{P_{s}:=\varphi_{s} P_{0} \varphi_{s}^{-1}: D_{\max } \longrightarrow \operatorname{ker} A_{s}^{*}\right\}
$$

is a continuous family of projections onto the solution spaces ker $A_{s}^{*}$. The projections are not necessarily orthogonal, but can be orthogonalized and remain continuous in $s$ like in [10. Lemma 12.8]. This proves the continuity of the family $\left\{\operatorname{ker} A_{s}^{*}\right\}$ in $D_{\max }$. 
Now we must show that $\left\{\gamma\left(\operatorname{ker} A_{s}^{*}\right)\right\}$ is a continuous family in the quotient space $D_{\max } / D_{m}$. This is not proved by the formula $\gamma\left(\operatorname{ker} A_{s}^{*}\right)=$ $\gamma\left(\varphi_{s}\left(\operatorname{ker} A_{0}^{*}\right)\right)$ alone. We must modify the endomorphism $\varphi_{s}$ of $D_{\max }$ in such a way that it keeps the subspace $D_{m}$ invariant.

By Proposition (3.6) , the Cauchy data space $\gamma\left(D_{m}+\operatorname{ker} A_{0}^{*}\right)$ is closed in $D_{\max } / D_{m}$. So $D_{m}+\operatorname{ker} A_{0}^{*}$ is closed in $D_{\max }$. We define a continuous family of mappings by

$$
\begin{aligned}
& \psi_{s}: D_{\max }=D_{m}+\operatorname{ker} A_{0}^{*}+\left(D_{m}+\operatorname{ker} A_{0}^{*}\right)^{\perp} \quad \longrightarrow \quad D_{\max } \\
& \begin{array}{lllll}
x+y & + & b & &
\end{array}
\end{aligned}
$$

with $\psi_{0}=$ id. Hence all $\psi_{s}$ are invertible for $s \ll 1$, and $\psi_{s}\left(D_{m}\right)=D_{m}$ for such small $s$. Hence we obtain a continuous family of mappings $\left\{\widetilde{\psi}_{s}: D_{\max } / D_{m} \rightarrow D_{\max } / D_{m}\right\}$ with $\widetilde{\psi}_{s}\left(\gamma\left(\operatorname{ker} A_{0}^{*}\right)\right)=\gamma\left(\operatorname{ker} A_{s}^{*}\right)$. From that we obtain a continuous family of projections as above.

REMARK 3.9. From the preceding arguments it also follows that the Cauchy data spaces form a differentiable family, if $\left\{A_{s}^{*}\right\}$ is a differentiable family.

3.4. Proof of the spectral flow formula. We begin with a simple case.

Lemma 3.10. Let $X$ be a Hilbert space, and $A \in \mathcal{C}(X)$ be a symmetric operator with $\operatorname{dom}(A)=D_{m}$ and $\operatorname{dom}\left(A^{*}\right)=D_{\max }$. Let $A_{D}:=\left.A^{*}\right|_{D}$ be a self-adjoint Fredholm extension of $A$. We assume that $A$ satisfies weak inner UCP. Then there exists an $\epsilon>0$ such that $A_{D}+a I$ is Fredholm and satisfies weak inner UCP for each $a \in[0, \epsilon]$. Let $\gamma: D_{\max } \rightarrow D_{\max } / D_{m}$ be the natural projection. Then we have

$$
\operatorname{sf}\left\{A_{D}+a I ; a \in[0, \epsilon]\right\}=-\operatorname{Mas}\left\{\gamma(D), \gamma\left(\operatorname{ker}\left(A^{*}+a I\right)\right) ; a \in[0, \epsilon]\right\} .
$$

ProOF. By the definition of the spectral flow we have

$$
\operatorname{sf}\left\{A_{D}+a I ; a \in[0, \epsilon]\right\}=\sum_{a \in(0, \epsilon]} \operatorname{dim} \operatorname{ker}\left(A_{D}+a I\right) .
$$

Let $\omega$ be the Green form on $D_{\max }$ induced by $A^{*}$. Let $W \in$ $\mathcal{L}\left(D_{\max } / D_{m}\right)$ be a Lagrangian complement of $\gamma\left(\operatorname{ker}\left(A^{*}+a_{0} I\right)\right)$. By Proposition [3.8, $\gamma\left(\operatorname{ker}\left(A^{*}+a I\right)\right)$ and $\operatorname{ker}\left(A^{*}+a I\right)$ are two differentiable families. For each $y\left(a_{0}\right) \in \operatorname{ker}\left(A_{D}+a_{0} I\right)$, there exists a continuous family $w(a) \in W+D_{m},\left|a-a_{0}\right|$ small, such that $w\left(a_{0}\right)=0$ and $y(a):=y\left(a_{0}\right)+w(a) \in \operatorname{ker}\left(A^{*}+a I\right)$. Since $A^{*}(y(a))=-a y(a)$ and the family $\{y(a)\}$ is continuous in $D_{\max }$, the family $\{y(a)\}$ is also 
continuous in $X$. For all $x\left(a_{0}\right) \in \operatorname{ker}\left(A_{D}+a_{0} I\right)$, we have

$$
\begin{aligned}
& \omega\left(\gamma\left(x\left(a_{0}\right)\right), \gamma(w(a))\right. \\
= & \left\langle A^{*}\left(x\left(a_{0}\right)\right), y(a)-y\left(a_{0}\right)\right\rangle-\left\langle x\left(a_{0}\right), A^{*}(w(a))\right\rangle \\
= & \left\langle-a_{0} x\left(a_{0}\right), y(a)-y\left(a_{0}\right)\right\rangle-\left\langle x\left(a_{0}\right), A^{*}(y(a))-A^{*}\left(y\left(a_{0}\right)\right)\right\rangle \\
= & \left\langle-a_{0} x\left(a_{0}\right), y(a)-y\left(a_{0}\right)\right\rangle-\left\langle x\left(a_{0}\right),-a y(a)+a_{0} y\left(a_{0}\right)\right\rangle \\
= & \left(a-a_{0}\right)\left\langle x\left(a_{0}\right), y(a)\right\rangle
\end{aligned}
$$

Let the crossing forms $Q$ and $\Gamma$ be defined by (2.19) and (2.20) respectively. Then we have $Q\left(\gamma\left(\operatorname{ker}\left(A^{*}+a I\right)\right), a_{0}\right)\left(\gamma\left(x\left(a_{0}\right)\right), \gamma\left(y\left(a_{0}\right)\right)=\right.$ $\left\langle x\left(a_{0}\right), y\left(a_{0}\right)\right\rangle$ and

$$
\Gamma\left(\gamma(D), \gamma\left(\operatorname{ker}\left(A^{*}+a I\right)\right), a_{0}\right)\left(\gamma\left(x\left(a_{0}\right)\right), \gamma\left(y\left(a_{0}\right)\right)=-\left\langle x\left(a_{0}\right), y\left(a_{0}\right)\right\rangle .\right.
$$

By Proposition 2.14 we have

$$
\begin{aligned}
\operatorname{Mas}\left\{\gamma(D), \gamma\left(\operatorname{ker}\left(A^{*}+a I\right)\right) ; a\right. & \in[0, \epsilon]\} \\
& =-\sum_{a \in(0, \epsilon]} \operatorname{dim} \operatorname{ker}\left(A_{D}+a I\right) .
\end{aligned}
$$

Combine equations (3.3), (3.4), and our lemma follows.

Now our main result follows at once.

Proof of Theorem 1.5. By Lemma 3.2. for each $s_{0}$ there exists an $\epsilon\left(s_{0}\right)>0$ such that the operators $A_{s}+a I$ satisfy weak inner UCP for all $s, a$ with $\left|s-s_{0}\right|,|a|<\epsilon\left(s_{0}\right)$. Here we use the continuity of the family $\left\{A_{s}^{*}\right\}$ as bounded operators from $D_{\max }$ to $X$. Since $[0,1]$ is compact and $A_{s, D_{s}}$ are Fredholm operators for all $s \in[0,1]$, there exists an $\epsilon>0$ such that the operators $A_{s}+a I$ satisfy weak inner UCP and $A_{s, D_{s}}+a I$ are Fredholm operators for all $s \in[0,1]$ and $|a|<\epsilon$.

We only need to prove the formula (1.12) in a small interval $\left[s_{0}, s_{1}\right]$. We consider the two-parameter families

$$
\left\{A_{s, D_{s}}+a I\right\} \text { and }\left\{\gamma\left(D_{s}\right), \gamma\left(\operatorname{ker} A_{s}^{*}+a I\right)\right\}
$$

for $s \in\left[s_{0}, s_{1}\right]$ and $a \in[0, \epsilon]$. Because of the homotopy invariance of spectral flow and Maslov index, both integers must vanish for the boundary loop going counter clockwise around the rectangular domain from the corner point $\left(s_{0}, 0\right)$ via the corner points $\left(s_{1}, 0\right),\left(s_{1}, \epsilon\right)$, and $\left(s_{0}, \epsilon\right)$ back to $\left(s_{0}, 0\right)$.

Moreover, for $s_{1}$ sufficiently close to $s_{0}$ we can choose $\epsilon$ sufficiently small so that $\operatorname{ker}\left(A_{s, D_{s}}+\epsilon I\right)=\{0\}$ for all $s \in\left[s_{0}, s_{1}\right]$. Hence, spectral flow and Maslov index must vanish on the top segment of our box. 
Finally, by the preceding lemma, the left and the right side segments of our curves yield vanishing sum of spectral flow and Maslov index. So, by additivity under catenation, our assertion follows.

\section{References}

[1] W. Ambrose, The index theorem in Riemannian geometry, Ann. of Math. 73 (1961), 49-86.

[2] V.I. ARNOL'D, Characteristic class entering in quantization conditions, Funkcional. Anal. i Priložen. 1 (1967), 1-14. (Russian; English translation: Functional Anal. Appl. 1 (1967), 1-13; French translation: Complément 1 to V.P. Maslov, "Théorie des perturbations et méthodes asymptotiques", Dunod, Gauthier-Villars, Paris, 1972, 341-361).

[3] M.F. Atiyah, V.K. Patodi, and I.M. Singer, Spectral asymmetry and Riemannian geometry. I, Math. Proc. Cambridge Phil. Soc. 77 (1975), 43-69.

[4] B. Bojarski, The abstract linear conjugation problem and Fredholm pairs of subspaces, in: "In Memoriam I.N. Vekua", Tbilisi Univ, Tbilisi, 1979, pp. 45-60 (Russian).

[5] B. Booss-Bavnbek and K. Furutani, The Maslov index - a functional analytical definition and the spectral flow formula, Tokyo J. Math. 21 (1998), $1-34$

[6] B. Booss-Bavnbek, K. Furutani, and N. Otsuki, Criss-cross reduction of the Maslov index and a proof of the Yoshida-Nicolaescu Theorem, Tokyo J. Math. 24 (2001), 113-128.

[7] B. Booss-Bavnbek, M. Lesch, and J. Phillips, Unbounded Fredholm operators and spectral flow, Canad. J. Math. 57/2 (2005), 225-250, arXiv: math.FA/0108014.

[8] B. Booss-Bavnbek, M. Lesch, And C. Zhu, Basic Analytic and Topological Properties of Formally Self-Adjoint Elliptic Differential Operators of First Order on Compact Manifolds with Smooth Boundary, in preparation.

[9] B. Booss-Bavnbek, M. Marcolli, and B.-L. Wang, Weak UCP and perturbed monopole equations, Internat. J. Math. 13/9 (2002), 987-1008.

[10] B. Booss-Bavnbek And K.P. WoJciechowski, Elliptic Boundary Problems for Dirac Operators, Birkhäuser, Boston, 1993.

[11] B. Booss-BAvnBeK And C. ZHu, Weak Symplectic Functional Analysis and General Spectral Flow Formula, Preprint, Roskilde, December 2003, arXiv: math.DG/0406139.

[12] S.E. Cappell, R. Lee, and E.Y. Miller, Selfadjoint elliptic operators and manifold decompositions Part II: Spectral flow and Maslov index, Comm. Pure Appl. Math. 49 (1996), 869-909.

[13] A. Carey and J. Phillips, Spectral Flow in Fredholm Modules, Eta Invariants and the JLO Cocycle, Preprint 2003, arXiv: math.KT/0308161.

[14] J.J. Duistermant, On the Morse index in variational calculus, Adv. Math. 21 (1976), 173-195.

[15] A. Floer, A relative Morse index for the symplectic action, Comm. Pure Appl. Math. 41 (1988), 393-407. 
[16] B. Himpel, P. Kirk, And M. Lesch, Calderón projector for the Hessian of the Chern-Simons function on a 3-manifold with boundary, Proc. London Math. Soc. (3) 89 (2004), 241-272, arXiv: math.GT/0302234.

[17] T. Kato, Perturbation Theory for Linear Operators, Springer-Verlag, Berlin, 1966, 2d ed., 1976, corrected printing, 1980.

[18] P. Kirk And M. Lesch, The $\eta$-invariant, Maslov index, and spectral flow for Dirac-type operators on manifolds with boundary, Forum Math. 16/4 (2004), 553-629, arXiv: math.DG/0012123.

[19] B. Lawruk, J. Śniatycki, AND W.M. Tulczyjew, Special symplectic spaces, J. Differential Equations 17 (1975), 477-497.

[20] Y. LONG AND C. ZHU, Maslov-type index theory for symplectic paths and spectral flow (II). Chinese Ann. of Math. 21B:1 (2000), 89-108.

[21] M. Monse, The Calculus of Variations in the Large, A.M.S. Coll. Publ., Vol.18, Amer. Math. Soc., New York, 1934.

[22] L. Nicolaescu, The Maslov index, the spectral flow, and decomposition of manifolds, Duke Math. J. 80 (1995), 485-533.

[23] J. Phillips, Self-adjoint Fredholm operators and spectral flow, Canad. Math. Bull. 39 (1996), 460-467.

[24] P. Piccione and D.V. Tausk, The Maslov index and a generalized Morse index theorem for non-positive definite metrics, C. R. Acad. Sci. Paris Sér. I Math. 331 (2000), 385-389.

[25] —, 一, The Morse index theorem in semi-Riemannian Geometry, Topology 41 (2002), 1123-1159, arXiv: math.DG/0011090

[26] A. PLIŚ, A smooth linear elliptic differential equation without any solution in a sphere, Comm. Pure Appl. Math. 14 (1961), 599-617.

[27] J. Robbin and D. Salamon, The Maslov index for paths, Topology 32 (1993), $827-844$.

[28] R.T. SeEley, Singular integrals and boundary value problems, Amer. J. Math. 88 (1966), 781-809.

[29] K.P. Wojciechowski, Spectral flow and the general linear conjugation problem, Simon Stevin 59 (1985), 59-91.

[30] Tomoyoshi Yoshida, Floer homology and splittings of manifolds, Ann. of Math. 134 (1991), 277-323.

[31] C. ZHU, Maslov-type index theory and closed characteristics on compact convex hypersurfaces in $\mathbb{R}^{2 n}, \mathrm{PhD}$ Thesis (in Chinese), Nankai Institute, Tianjin, 2000.

[32] C. ZHu, The Morse Index Theorem for Regular Lagrangian Systems, Preprint September 2001 (arXiv: math.DG/0109117) (first version); MPI Preprint 2003, no. 55 (modified version).

[33] C. Zhu And Y. Long, Maslov-type index theory for symplectic paths and spectral flow. (I), Chinese Ann. of Math. 20B (1999), 413-424. 
Institut FOR MATEMATIK OG FYSIK, ROSKILDE UNIVERSiTy, 4000 RosKILDE, DENMARK

E-mail address: booss@mmf.ruc.dk

$U R L:$ http://imfufa.ruc.dk/ Booss

Nankai Institute of Mathematics, Key Lab of Pure Mathematics and Combinatorics of Ministry of Education, Nankai University, TianJin 300071, People's Republic of China

E-mail address: zhucf@nankai.edu.cn 